\title{
Novel mechanism of angiotensin II-induced cardiac injury in hypertensive rats: the critical role of ASK1 and VEGF
}

\author{
Hisato Nako ${ }^{1}$, Keiichiro Kataoka ${ }^{1}$, Nobutaka Koibuchi ${ }^{1}$, Yi-Fei Dong ${ }^{1}$, Kensuke Toyama ${ }^{1}$, Eiichiro Yamamoto $^{2}$, \\ Osamu Yasuda ${ }^{2}$, Hidenori Ichijo ${ }^{3}$, Hisao Ogawa ${ }^{4}$ and Shokei Kim-Mitsuyama ${ }^{1}$
}

This study was undertaken to elucidate a novel mechanism underlying angiotensin II-induced cardiac injury, focusing on the role of oxidative stress and myocardial capillary density. Salt-loaded Dahl salt-sensitive hypertensive rats (DS rats), a useful model for hypertensive cardiac remodeling or heart failure, were orally given irbesartan (an AT1 receptor blocker), tempol (a superoxide dismutase mimetic) or hydralazine (a vasodilator). Irbesartan significantly ameliorated left ventricular ischemia and prevented the development of cardiac hypertrophy and fibrosis in DS rats. The benefits were associated with the attenuation of oxidative stress, normalization of myocardial capillary density and inhibition of capillary endothelial apoptosis. Moreover, DS rats with significant cardiac hypertrophy and fibrosis displayed decreased myocardial vascular endothelial growth factor (VEGF) expression and increased cardiac apoptosis signal-regulating kinase 1 (ASK1) activation. Treatment with irbesartan significantly reversed these phenotypes. Tempol treatment of DS rats mimicked all the above-mentioned effects of irbesartan, indicating the critical role of oxidative stress in cardiac injury. We also investigated the role of VEGF and ASK1 in oxidative stress-induced endothelial apoptosis by using cultured endothelial cells from wild-type and ASK1-deficient mice. Oxidative stress-induced ASK1 activation led to endothelial apoptosis, and VEGF treatment prevented oxidative stress-induced endothelial apoptosis by inhibiting ASK1 activation. We obtained the first evidence that oxidative stress-induced cardiac VEGF repression and ASK1 activation caused the enhancement of endothelial apoptosis and contributed to a decrease in myocardial capillary density. These effects resulted in angiotensin II-induced progression of cardiac injury.

Hypertension Research (2012) 35, 194-200; doi:10.1038/hr.2011.175; published online 17 November 2011

Keywords: ASK1; endothelial apoptosis; heart failure; oxidative stress; VEGF

\section{INTRODUCTION}

Accumulating evidence indicates that angiotensin II has a key role in the transition from compensated to decompensated cardiac hypertrophy or heart failure. ${ }^{1-4}$ Clinical evidence shows that reninangiotensin system blockers, such as AT1 receptor blockers or angiotensin-converting enzyme inhibitors, are effective treatments for both cardiac hypertrophy and decompensated cardiac hypertrophy or heart failure. ${ }^{5,6}$ However, the precise mechanism underlying the protective effects of renin-angiotensin system blockers against decompensated cardiac hypertrophy is not entirely clear.

Emerging evidence shows that the progression of decompensated cardiac hypertrophy is influenced by abnormalities in cardiac myocytes and myocardial ischemias that can be attributed to a reduction in myocardial capillary density. ${ }^{7-10}$ Therefore, recent work has focused on examining the regulatory mechanisms of capillary density in cardiac hypertrophy. However, the role of angiotensin II in the regulation of capillary density during cardiac hypertrophy is still unclear.

In this study, we examined the effects of an AT1 receptor blocker on myocardial capillary density and cardiac ischemia in Dahl salt-sensitive hypertensive rats (DS rats) to elucidate the novel mechanism of angiotensin II-induced cardiac injury. Dahl saltsensitive rat is a useful model for hypertensive decompensated cardiac hypertrophy and heart failure. We obtained evidence that the prevention of cardiac injury by angiotensin II blockades could be partially attributed to the amelioration of cardiac ischemia. This beneficial effect was mediated by the inhibition of downregulation of the oxidative stress-induced myocardial vascular endothelial growth factor (VEGF) and activation of apoptosis signal-regulating kinase 1 (ASK1).

\footnotetext{
${ }^{1}$ Department of Pharmacology and Molecular Therapeutics, Kumamoto University Graduate School of Medical Sciences, Kumamoto, Japan; ${ }^{2}$ Department of Cardiovascular Clinical and Translational Research, Kumamoto University Hospital, Kumamoto, Japan; ${ }^{3}$ Laboratory of Cell Signaling, Tokyo University Graduate School of Pharmaceutical Sciences, Tokyo, Japan and ${ }^{4}$ Department of Cardiovascular Medicine, Kumamoto University Graduate School of Medical Sciences, Kumamoto, Japan

Correspondence: Dr S Kim-Mitsuyama, Department of Pharmacology and Molecular Therapeutics, Kumamoto University Graduate School of Medical Sciences, 1-1-1 Honjyo, Kumamoto 860-8556, Japan.

E-mail. kimmitsu@gpo.kumamoto-u.ac.jp

Received 13 January 2011; revised 25 July 2011; accepted 26 July 2011; published online 17 November 2011
} 


\section{METHODS}

Animals

All procedures were in accordance with institutional guidelines for animal research. DS rats (Japan SLC, Inc., Shizuoka, Japan) were used in this study.

\section{Treatment of salt-loaded DS rats with irbesartan, tempol and hydralazine}

At 7 weeks of age, the diet of DS rats was switched from a $0.3 \% \mathrm{NaCl}$ (low-salt) diet to an $8 \% \mathrm{NaCl}$ (high-salt) diet. Previously, we ${ }^{2-4}$ and other groups ${ }^{11}$ demonstrated that 12-week-old DS rats display compensated cardiac hypertrophy with normal cardiac function. Therefore, drug treatment in the DS rats was initiated at 12 weeks of age. DS rats (12-week-old) were given irbesartan $\left(20 \mathrm{mg} \mathrm{kg}^{-1} \mathrm{day}^{-1}\right)$, tempol $\left(0.1 \mathrm{mmol} \mathrm{kg}^{-1} \mathrm{day}^{-1}\right)$ or hydralazine $\left(3 \mathrm{mg} \mathrm{kg}^{-1} \mathrm{day}^{-1}\right)$ orally for 4 weeks (from 12 to 16 weeks of age). In preliminary experiments, we found that irbesartan, tempol and hydralazine at the above-mentioned doses exerted similar hypotensive effects in DS rats. Therefore, this experimental protocol allowed us to compare the effects of each drug under the same blood pressure control. Blood pressure was measured with the tail-cuff method every week. Echocardiographic assessment was performed biweekly during the drug treatment. After 4 weeks of treatment, DS rats were anesthetized with ether and the hearts were immediately excised to examine various parameters, as described below.

\section{Measurement of cardiac ischemia}

Cardiac hypoxia was estimated with Hypoxyprobe ${ }^{\mathrm{TM}}-1$ (Hypoxyprobe, Burlington, MA, USA) according to the manufacturer's instructions. In brief, Hypoxyprobe $^{\mathrm{TM}}-1$ (60 mg per kg body weight) was intraperitoneally injected into DS rats. The animal was anesthetized with ether, $15 \mathrm{~min}$ after the injection. The heart was then immediately perfused with phosphate-buffered saline and removed. Pimonidazole, which is an element of Hypoxyprobe ${ }^{\mathrm{TM}}-1$ that is reductively activated in hypoxic cells, was stained with DAB (3'3-diaminobenzidine) (DAKO, Glostrop, Denmark). Pimonidazole-positive areas were quantified using the Lumina Vision version 2.2 analysis software (Mitani Corporation, Tokyo, Japan) from 10 sections taken at $\times 200$ magnification.

\section{Measurement of capillary density}

For assessment of cardiac capillary density, cross-sectional heart samples were immunostained with an anti-CD31 antibody (Santa Cruz Biotechnology, Tokyo, Japan) (working dilution 1:200). The number of cardiac CD31-positive cells was counted in 10 fields that were taken at $\times 200$ magnification for each individual rat. The average CD31-positive cell number was obtained for each rat. Capillary density was expressed as the average number of capillaries per cardiomyocyte.

\section{Measurement of capillary apoptosis}

Cardiac capillary apoptosis of DS rats was detected with the TdT-mediated dUTP nick end-labeling (TUNEL) assay by utilizing an in situ apoptosis detection kit (TaKaRa Bio Inc., Shiga, Japan) in combination with immunostaining using the anti-CD31 antibody (Santa Cruz). The number of cardiac TUNEL and CD31 double-positive cells and CD31-positive cells was counted in 10 fields that were taken at $\times 200$ magnification from individual rats. The average ratio of TUNEL and CD31 double-positive cells to CD31-positive cells was obtained for each individual rat.

\section{Measurement of cardiac superoxide}

Cardiac tissue that was removed from DS rats was immediately frozen in Tissue-Tek O.C.T embedding medium (Sakura Finetek, Tokyo, Japan). Dihydroethidium was used to evaluate the levels of cardiac and vascular superoxide in situ, as described previously. ${ }^{12}$

\section{Western blot analysis}

Western blotting was performed as described previously. ${ }^{13}$ Antibodies used were as follows: anti-VEGF $(2000 \times$, Santa Cruz) and anti-phosphorylated ASK1 $(1000 \times) .{ }^{14}$ The intensity of the bands was quantified using the NIH Image analysis software v1.61 (National Institutes of Health, Bethesda, MD, USA). In individual samples, each value was corrected against the $\alpha$-tubulin levels.
In vitro experiments on cultured endothelial cells

Aortic endothelial cells were isolated using the EasySep Mouse PE selection kit (STEMCELL, Tokyo, Japan) from male ASK1-/- mice ${ }^{15}$ and wild-type mice (C57BL/6J). Endothelial cells were grown using the EGM-2 BulletKit (Lonza, Basel, Switzerland) and used between passages four and six.

To examine the role of ASK1 and VEGF in $\mathrm{H}_{2} \mathrm{O}_{2}$-induced endothelial apoptosis, sub-confluent endothelial cells were starved overnight in EBM-2 media (Lonza) containing $1.2 \%$ serum in the absence or presence of $2 \mathrm{ng} \mathrm{ml}^{-1}$ $\operatorname{VEGF}_{164}(\mathrm{R} \& \mathrm{D}$, Tokyo, Japan) and then incubated for $24 \mathrm{~h}$ in starvation medium supplemented with $\mathrm{H}_{2} \mathrm{O}_{2}(200 \mu \mathrm{M})$. To assess the apoptosis, endothelial cells were immunostained with an anti-active caspase 3 antibody (R\&D) (working dilution 1:200). The number of active caspase 3-positive cells was counted in 10 fields that were taken at $\times 400$ magnification. Each field contained between 300 and 400 endothelial cells.

To examine the role of VEGF in $\mathrm{H}_{2} \mathrm{O}_{2}$-induced endothelial ASK1 activation, subconfluent endothelial cells were starved overnight in EBM-2 containing $1.2 \%$ serum in the absence or presence of $2 \mathrm{ng} \mathrm{ml}^{-1} \mathrm{VEGF}_{164}$, and then incubated for 5 or $30 \mathrm{~min}$ in starvation medium supplemented with $\mathrm{H}_{2} \mathrm{O}_{2}$ $(200 \mu \mathrm{M})$. To assess the ASK1 activation in endothelial cells, western blot analysis was performed, as described above.

\section{Measurement of intracellular reactive oxygen species in vitro}

The determination of intracellular oxidative stress formation was based on the oxidation of $2^{\prime}, 7^{\prime}$-dichlorofluorescin diacetate (H2DCFDA, Sigma-Aldrich Corporation, St Louis, MO, USA), which induces the production of an intracellular fluorescent compound. After $24 \mathrm{~h}$ of starvation with or without $\operatorname{VEGF}_{164}\left(2 \mathrm{ng} \mathrm{ml}^{-1}\right)$, endothelial cells were pre-loaded with H2DCFDA at a final concentration of $10 \mu \mathrm{M}$ for $15 \mathrm{~min}$. Then, $\mathrm{H}_{2} \mathrm{O}_{2}$ was added at a final concentration of $200 \mu \mathrm{M}$ for $5 \mathrm{~min}$, after which the medium was removed and replaced with phosphate-buffered saline. Fluorescent images were captured with a fluorescence microscope and the fluorescence intensity was quantitated with image analysis software (Lumina Vision).

\section{Echocardiographic assessment}

Transthoracic echocardiographic studies were performed with an echocardiographic system equipped with a $12-\mathrm{MHz}$ echocardiographic probe (PHILIPS SONOS-4500, Tokyo, Japan), as previously described. ${ }^{2,4}$ In brief, DS rats were lightly anesthetized with an intraperitoneal administration of ketamine $\mathrm{HCl}$ $\left(50 \mathrm{mg} \mathrm{kg}^{-1}\right)$ and xylazine $\mathrm{HCl}\left(10 \mathrm{mg} \mathrm{kg}^{-1}\right)$, and then held in the half leftlateral position. M-mode tracings were recorded through the left ventricular (LV) anterior and posterior walls at the papillary muscle level to measure LV end-diastolic dimension, LV end-systolic dimension, fractional shortening, LV ejection fraction, LV anterior wall thickness at end diastole and posterior wall thickness at end diastole.

\section{RT-PCR}

Total RNA was prepared using TRIzol reagent (Invitrogen, Tokyo, Japan) from the LV tissue. Complementary DNA were synthesized by standard techniques, using the QuantiTect Reverse Transcription Kit (QIAGEN Inc., Hilden, Germany). Real time PCR reactions were performed, recorded and analyzed using the Thermal Cycler Dice Real Time System (TaKaRa Bio Inc., Shiga, Japan) with SYBR Green I detection, as described previously. ${ }^{13}$ Complementary DNA was amplified using the SYBR Premix Ex TaqTM (Perfect Real Time) PCR kit (TaKaRa Bio Inc.) with specific BNP primers: primer forward: $5^{\prime}$ GTCTCCAGAACAATCCACGATG-3': and primer reverse: 5'-AAGGCGCT GTCTTGAGACCTAA-3'.

\section{Histological analysis and immunohistochemistry}

All hearts were fixed overnight with $4 \%$ formalin, embedded in paraffin, cut into $4 \mu \mathrm{m}$-thick coronal sections and stained with Sirius Red F3BA (0.5\% in saturated aqueous picric acid, (Sigma-Aldrich, St Louis, MO, USA) Aldrich Chemical Company) to assess cardiac interstitial fibrosis. The area of fibrosis was assessed by using Lumina Vision version 2.2 analysis software. ${ }^{16}$

To assess cardiac macrophage infiltration, the heart sections were immunostained with an anti-ED-1 antibody (BMA Biomedicals AG, Augst, Switzerland) (working dilution 1:500) for the identification of monocytes/macrophages, as previously described. ${ }^{16}$ The number of cardiac ED-1-positive cells was counted 
in 10 fields from individual rats. The average number of ED-1-positive cells was obtained for the individual rats.

\section{Statistical analysis}

All data are presented as the mean \pm s.e.m. The data on the time course experiments were analyzed by a two-way analysis of variance, followed by Fisher's PLSD test, using StatView for Windows (SAS Institute, Inc. Cary, NC, USA). For all other data, statistical significance was determined with one-way analysis of variances, followed by Fisher's PLSD tests. In all tests, differences were considered statistically significant at a value of $P<0.05$.

\section{RESULTS}

Effects on blood pressure and LV weight

As shown in Figure 1, irbesartan, tempol or hydralazine significantly and similarly reduced the blood pressure of DS rats throughout the treat-

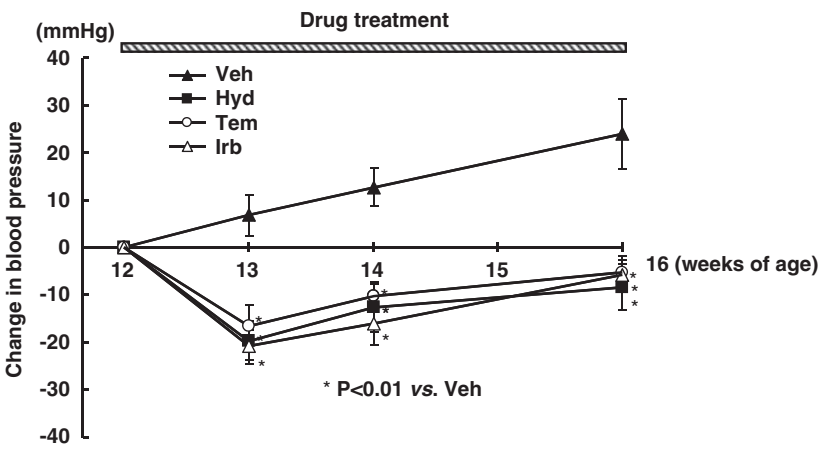

Figure 1 Effect of hydralazine, tempol and irbesartan on blood pressure of DS rats. Hyd, hydralazine-treated DS rats; Irb, irbesartan-treated DS rats; Tem, tempol-treated DS rats; Veh, vehicle-treated DS rats. Each value represents mean \pm s.e.m. ( $n=9$ in Veh, $n=7$ in Hyd, $n=7$ in Tem, $n=8$ in Irb). ment, and there were no significant differences in blood pressure with any of the drug treatments. As shown in Figure 2 and Table 1, despite comparable hypotensive effect, irbesartan or tempol reduced LV weight, LV BNP expression, macrophage infiltration and interstitial fibrosis in DS rats to a greater extent than treatment with hydralazine. Irbesartan and tempol significantly reduced the increase in lung weight in DS rats whereas hydralazine failed to reduce the increase in lung weight (Table 1).

As shown in Table 2, there was no significant difference in LV enddiastolic dimension, end-systolic dimension, ejection fraction, and fractional shortening between high-salt and low-salt fed DS rats, indicating that there was no apparent cardiac dysfunction in saltloaded DS rats in this study. Thus, the change of lung weight in the salt-loaded DS rats should be attributed to the increase in body fluid retention that is well known to occur in DS rats. The lung weight appears not to be related to the cardiac dysfunction.

\section{Effects on $\mathbf{L V}$ oxidative stress and myocardial ischemia}

As shown in Figure 3a, irbesartan or tempol, but not hydralazine, significantly prevented the increase in LV oxidative stress of DS rats. Interestingly, the significant reduction of LV superoxide by irbesartan or tempol was closely associated with a significant attenuation of LV ischemia, as shown by the decrease in the LV pimonidazole-positive area (Figure 3b). Hydralazine failed to reduce cardiac oxidative stress or the pimonidazole-positive area in DS rats.

We also measured cardiac endothelial nitric oxide synthase (eNOS) in each group of rats and found no significant differences between cardiac phospho-eNOS and total eNOS among each group of rats.

Effects on LV capillary density and capillary endothelial apoptosis As shown in Figure 4a, the cross-sectional area of cardiomyocytes in salt-loaded DS rats, which was significantly larger than in the control a

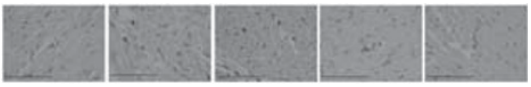

(counts $/ \mathrm{mm}^{2}$ )

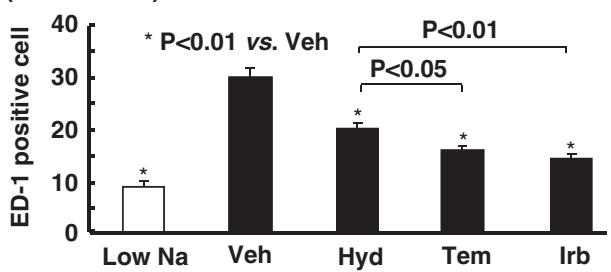

b
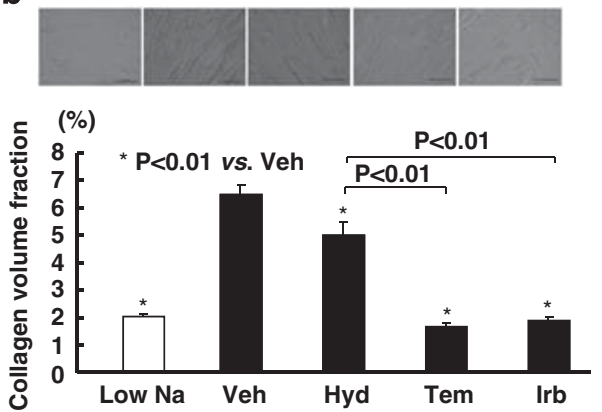

Figure 2 Effect of hydralazine, tempol and irbesartan on cardiac inflammation (a) and cardiac interstitial fibrosis (b) of DS rats. Upper panels in (a) and (b) indicate representative photomicrographs stained with ED1 and Sirius red, respectively. Low Na indicates low salt-fed DS rats. High salt-fed DS rats were orally given hydralazine (Hyd), tempol (Tem) or irbesartan (Irb) for 4 weeks. Values are means \pm s.e.m. ( $n=5$ in Low Na, $n=9$ in Veh, $n=7$ in Hyd, $n=7$ in Tem, $n=8$ in Irb). Bar $=100 \mu \mathrm{m}$. A full color version of this figure is available at the Hypertension Research journal online.

Table 1 Body weight, cardiac weight, cardiac BNP mRNA and lung weight in DS rats

High $\mathrm{Na}$

\begin{tabular}{|c|c|c|c|c|c|}
\hline & \multirow[b]{2}{*}{ Low $N a(n=5)$} & \\
\hline & & Veh $(n=9)$ & Hyd $(\mathrm{n}=7)$ & $\operatorname{Tem}(n=7)$ & $\operatorname{Irb}(\mathrm{n}=8)$ \\
\hline $\mathrm{BW}(\mathrm{g})$ & $400 \pm 10^{\#}$ & $376 \pm 6$ & $384 \pm 4$ & $405 \pm 6^{*}$ & $391 \pm 3^{\#}$ \\
\hline LV/BW(mg g $\left.{ }^{-1}\right)$ & $2.21 \pm 0.07^{\#}$ & $3.42 \pm 0.04$ & $3.25 \pm 0.03^{*}$ & $3.03 \pm 0.06^{\# \S}$ & $3.08 \pm 0.03 * \S$ \\
\hline BNP mRNA expression & $1.26 \pm 0.22^{\#}$ & $3.69 \pm 0.30$ & $3.51 \pm 0.32$ & $3.08 \pm 0.50 *$ & $2.65 \pm 0.48^{*}$ \\
\hline Lung/BW(mg g $\left.{ }^{-1}\right)$ & $3.42 \pm 0.04^{\#}$ & $4.31 \pm 0.08$ & $4.29 \pm 0.07$ & $3.94 \pm 0.07^{\#}$ & $3.89 \pm 0.05^{\#}$ \\
\hline
\end{tabular}

Abbreviations: BNP, brain natriuretic peptide; BW, body weight; LV, left ventricular weight.

Low Na indicates low salt-fed DS rats. High salt-fed DS rats were treated with vehicle (Veh), hydralazine (Hyd), tempol (Tem) or irbesartan (Irb) for 4 weeks.

Values are means \pm s.e.m. ${ }^{*} P<0.05,{ }^{\#} P<0.01$ vs. Veh. ${ }^{\$} P<0.01$ vs. Hyd. 
Table 2 Echocardiographic data in each group of DS rats

\begin{tabular}{|c|c|c|c|c|c|}
\hline & \multirow[b]{2}{*}{ Low $\mathrm{Na}(\mathrm{n}=9)$} & \multicolumn{4}{|c|}{ High $\mathrm{Na}$} \\
\hline & & $\operatorname{Veh}(n=12)$ & Hyd $(\mathrm{n}=9)$ & Tem $(\mathrm{n}=11)$ & $\operatorname{lrb}(\mathrm{n}=13)$ \\
\hline LVPw (mm) & $1.38 \pm 0.04^{\#}$ & $2.14 \pm 0.02$ & $1.95 \pm 0.03^{\#}$ & $1.78 \pm 0.04^{\# \S}$ & $1.77 \pm 0.03^{\# \S}$ \\
\hline LVDd (mm) & $8.67 \pm 0.12$ & $8.46 \pm 0.10$ & $8.46 \pm 0.26$ & $8.90 \pm 0.13$ & $8.79 \pm 0.20$ \\
\hline LVDd/BW (mm per $100 \mathrm{~g} \mathrm{BW}$ ) & $2.23 \pm 0.05$ & $2.44 \pm 0.11$ & $2.33 \pm 0.03$ & $2.47 \pm 0.11$ & $2.45 \pm 0.06$ \\
\hline $\mathrm{EF}(\%)$ & $69.8 \pm 2.46$ & $74.6 \pm 2.67$ & $73.7 \pm 3.99$ & $71.1 \pm 2.0$ & $73.7 \pm 2.46$ \\
\hline FS (\%) & $35.7 \pm 2.06$ & $40.2 \pm 2.67$ & $39.9 \pm 3.95$ & $35.9 \pm 1.46$ & $39.0 \pm 2.14$ \\
\hline
\end{tabular}

Abbreviations: BNP, brain natriuretic peptide; BW, body weight; EF, ejection fraction; FS, fractional shortening; LV, left ventricular weight; LVAw, left ventricular anterior wall thickness; LVDd, left ventricular end-diastolic dimension; LVDs, left ventricular end-systolic dimension; LVPw, left ventricular posterior wall thickness.

Values are means \pm s.e.m. $\# P<0.01$ vs. Veh. $\$ P<0.01$ vs. Hyd.

a

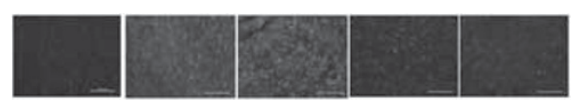

(\%)

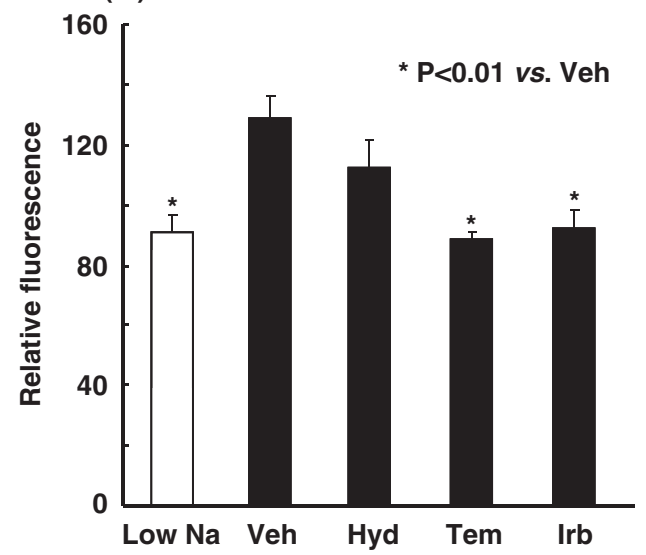

b

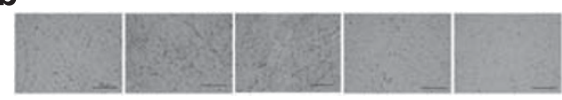

(\%)

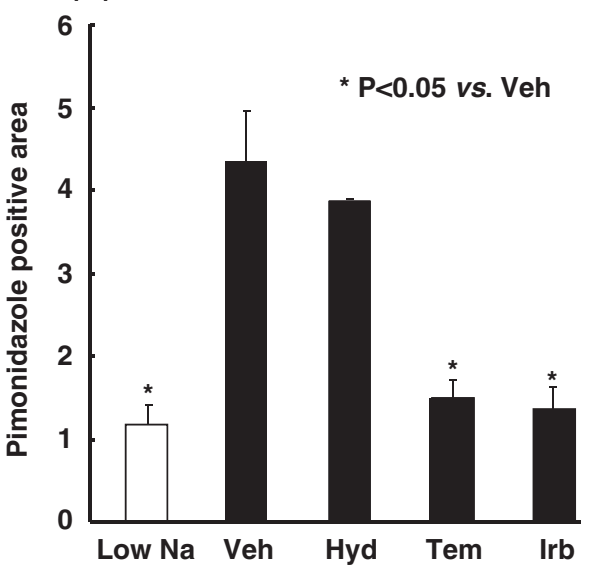

Figure 3 Effect of hydralazine, tempol and irbesartan on cardiac superoxide (a) and cardiac ischemia (b) of DS rats. Cardiac superoxide was detected with dihydroethidium staining as described in Materials and methods. Upper panels in (a) and (b) indicate representative photomicrographs stained with dihydroethidium and pimonidazole, respectively. Hyd, hydralazine-treated DS rats; Irb, irbesartan-treated DS rats; Tem, tempol-treated DS rats; Veh, vehicletreated DS rats. Values are means \pm s.e.m. ( $n=5$ in Low $\mathrm{Na}, n=9$ in Veh, $n=7$ in Hyd, $n=7$ in Tem, $n=8$ in Irb). Bar=100 $\mu$ m. A full color version of this figure is available at the Hypertension Research journal online.

DS rats, was significantly attenuated by irbesartan or tempol, but not hydralazine. As shown in Figure 4b, the LV capillary density in DS rats was significantly smaller than in the control DS rats (low salt DS rats), as shown by the number of LV CD31-positive cells per cardiomyocyte. Irbesartan or tempol treatment significantly and similarly prevented the decrease in the LV capillary density of DS rats. However, treatment with hydralazine did not significantly prevent this decrease.

As shown in Figure 4c, DS rats displayed an increase in LV capillary endothelial apoptosis when compared with the control DS rats, as shown by double staining for TUNEL and CD31. Interestingly, irbesartan or tempol, but not hydralazine, significantly prevented the increase in capillary endothelial apoptosis in DS rats.

However, 12-week-old DS rats with compensated cardiac hypertrophy had no decrease in LV capillary density and no increase in capillary endothelial apoptosis (data not shown).

\section{Effects on LV VEGF and ASK1}

As shown in Figure 5a, LV VEGF protein levels in DS rats were significantly less than in control DS rats. Irbesartan or tempol treatment significantly attenuated the decrease in LV VEGF protein levels in DS rats. However, hydralazine did not increase VEGF levels in DS rats.

As shown in Figure 5b, LV ASK1 phosphorylation in DS rats was significantly higher than that in control DS rats. Irbesartan or tempol significantly reduced LV ASK1 phosphorylation in DS rats to a greater extent than hydralazine treatment.

However, 12-week-old DS rats with compensated cardiac hypertrophy had no decrease in VEGF and no increase in LV ASK1 activation (data not shown).

\section{Role of ASK1 and VEGF in endothelial apoptosis}

As shown in Figure $6 \mathrm{a}, \mathrm{H}_{2} \mathrm{O}_{2}$ significantly increased vascular endothelial apoptosis, as shown by the increase in active caspase 3-positive cells. However, endothelial apoptosis induced by $\mathrm{H}_{2} \mathrm{O}_{2}$ was decreased in ASK1-deficient mice when compared with wild-type mice. As shown in Figure 6b, pretreatment with VEGF significantly suppressed $\mathrm{H}_{2} \mathrm{O}_{2}$-induced vascular endothelial cell apoptosis. As shown in Figure $6 \mathrm{c}, \mathrm{H}_{2} \mathrm{O}_{2}$ significantly activated endothelial ASK1 in the 


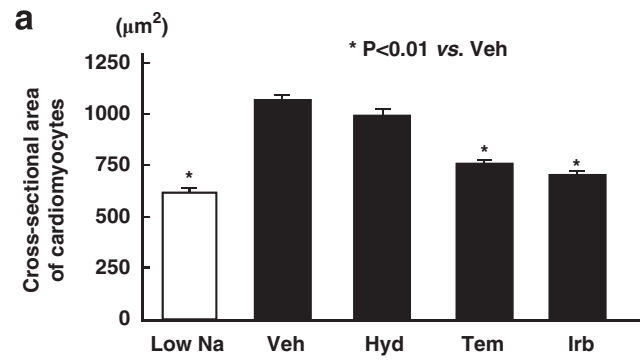

C
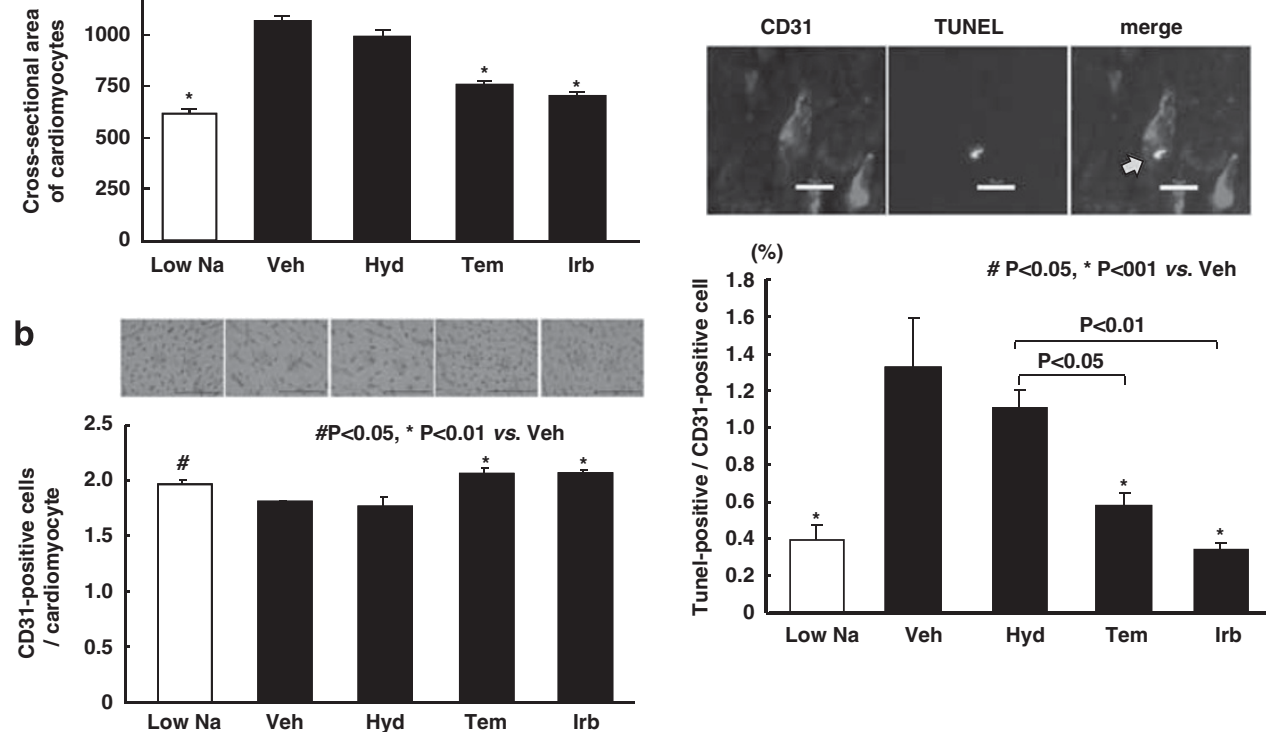

Figure 4 Effect of hydralazine, tempol and irbesartan on cross-sectional area of cardiomyocytes (a), cardiac capillary density (b) and capillary endothelial apoptosis (c) of DS rats. Upper panels in (b) and (c) indicate representative photomicrographs immunostained with CD31 antibody, and double stained with CD31 and TUNEL, respectively. Hyd, hydralazine-treated DS rats; Irb, irbesartan-treated DS rats; Tem, tempol-treated DS rats; Veh, vehicle-treated DS rats. Each value represents mean \pm s.e.m. ( $n=5$ in Low $\mathrm{Na}, n=9$ in Veh, $n=7$ in Hyd, $n=7$ in Tem, $n=8$ in Irb). Bar=100 $\mu$ m.A full color version of this figure is available at the Hypertension Research journal online.

a
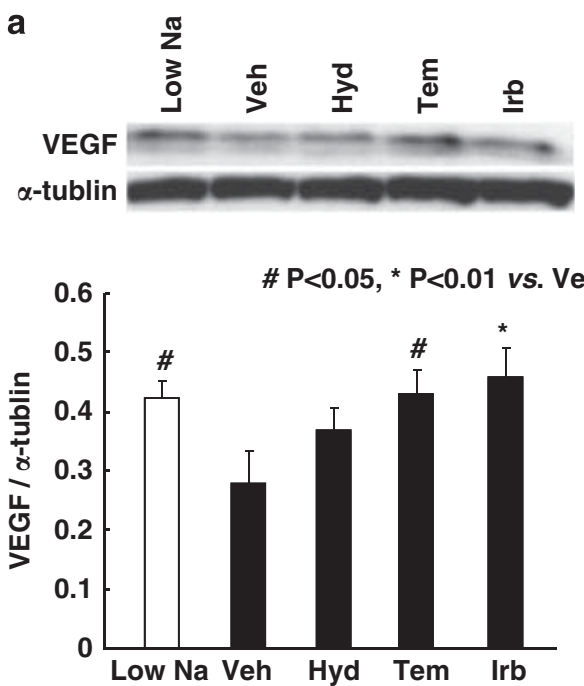

b
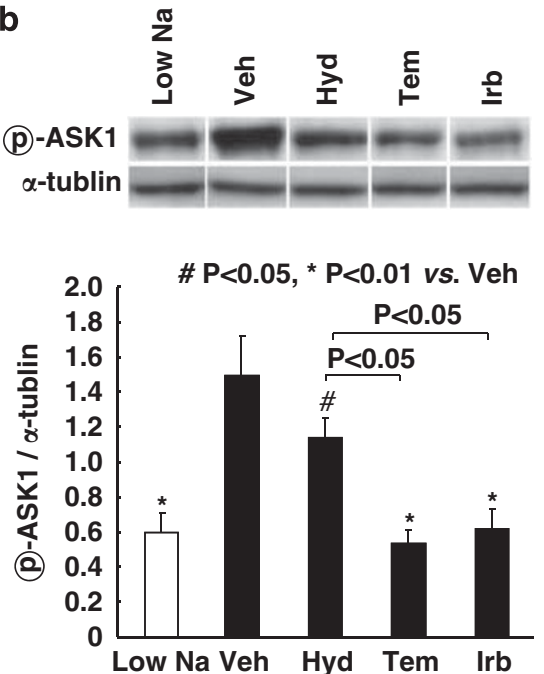

Figure 5 Effect of hydralazine, tempol and irbesartan on cardiacVEGF (a) and phospho-ASK1 (b) of DS rats. Upper panels in (a) and (b) indicate representative western blots of VEGF and phospho-ASK1, respectively. Hyd, hydralazine-treated DS rats; Irb, irbesartan-treated DS rats; Tem, tempol-treated DS rats; Veh, vehicle-treated DS rats. VEGF or phospho-ASK1 density in individual samples was corrected for $\alpha$-tubulin density. Each value represents mean \pm s.e.m. ( $n=5$ in Low $\mathrm{Na}, n=9$ in Veh, $n=7$ in Hyd, $n=7$ in Tem, $n=8$ in Irb).

wild-type mice. However, pretreatment with VEGF significantly prevented $\mathrm{H}_{2} \mathrm{O}_{2}$-induced activation of endothelial ASK1.

As shown in Figure 6d, VEGF treatment significantly attenuated $\mathrm{H}_{2} \mathrm{O}_{2}$-derived oxidative stress in endothelial cells $(P<0.01)$.

\section{DISCUSSION}

The major finding of this study is that the prevention of cardiac hypertrophy by angiotensin II blockade was accompanied by amelioration of LV ischemia because the reduction in myocardial capillary density was suppressed. These beneficial effects were mediated by a reduction in oxidative stress-induced endothelial apoptosis through normalization of VEGF and ASK1 levels. Thus, our present work provides a novel insight into the molecular mechanisms underlying angiotensin II-induced cardiac injury.

Compensated cardiac hypertrophy is associated with a normal or increased number of myocardial capillaries. ${ }^{7-10}$ However, prolonged cardiac hypertrophy leads to cardiac ischemia as a result of the reduction in myocardial capillary density. This reduction is one of 

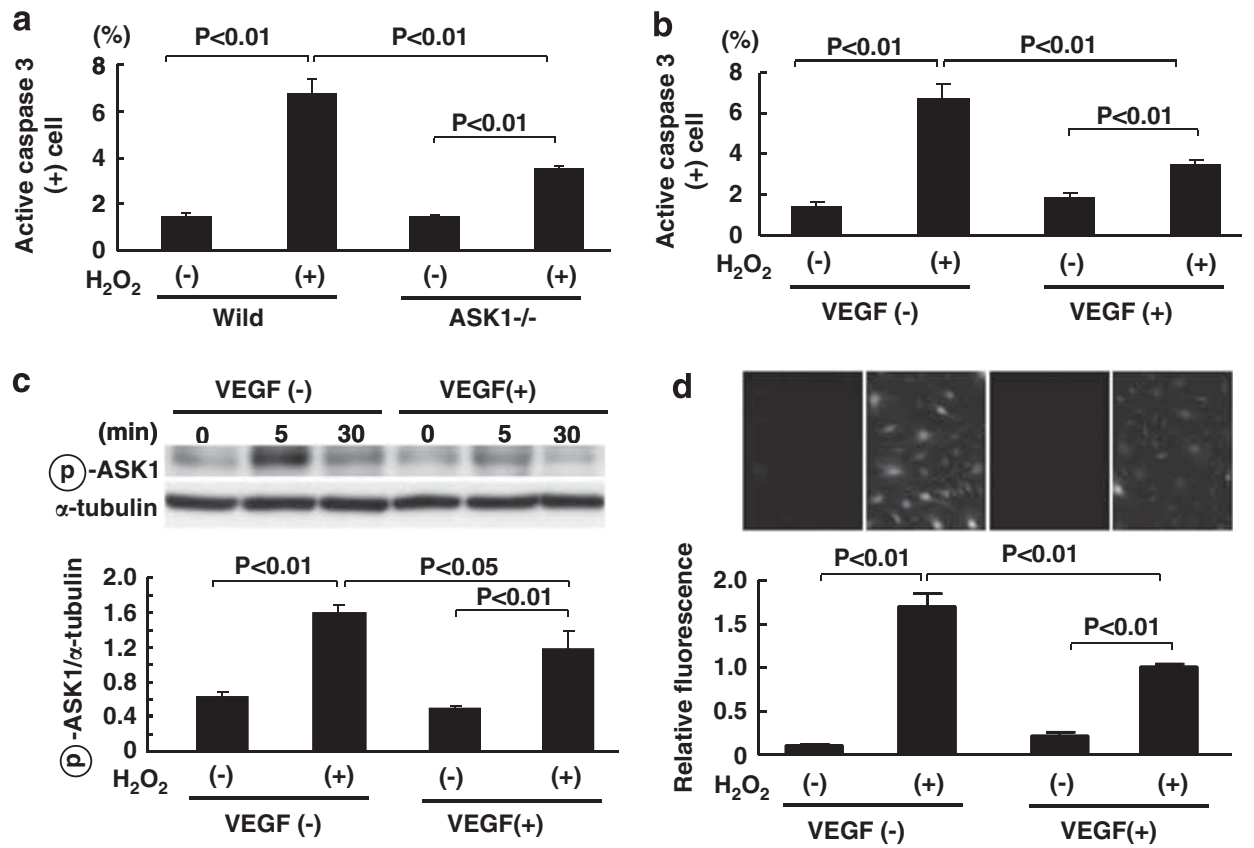

Figure 6 Role of ASK1 (a) and VEGF (b) in $\mathrm{H}_{2} \mathrm{O}_{2}$-induced endothelial apoptosis, and effect of VEGF on endothelial ASK1 activation (c) and endothelial oxidative stress (d). (a) Indicates comparison between $\mathrm{H}_{2} \mathrm{O}_{2}$-induced endothelial apoptosis from wild-type and ASK1-/- mice. (b) Indicates the effect of VEGF on $\mathrm{H}_{2} \mathrm{O}_{2}$-induced endothelial apoptosis from wild-type mice. (c) Indicates the effect of VEGF on $\mathrm{H}_{2} \mathrm{O}_{2}$-induced endothelial ASK1 activation from wildtype mice. (d) Upper panels indicate representative photomicrographs of fluorescent images in each group. Each value represents mean \pm s.e.m. $(n=5$ in each group).A full color version of this figure is available at the Hypertension Research journal online.

the main mechanisms that is responsible for the transition from compensated to decompensated cardiac hypertrophy. ${ }^{7-10}$ Blocking VEGF leads to a decrease in myocardial capillary density in pressure overload-induced cardiac hypertrophy models, resulting in the transition from compensated to decompensated cardiac hypertrophy and thus leading to the development of heart failure. ${ }^{10}$ Moreover, pressure overload-induced cardiac hypertrophy in VEGF-deficient mice leads to a reduction in myocardial capillary density, which then accelerates the transition from compensated myocardial hypertrophy to heart failure. ${ }^{17}$ Thus, myocardial VEGF plays a protective role against the development of decompensated cardiac hypertrophy. However, the potential contribution of angiotensin II to the regulation of capillary density in cardiac hypertrophy remains to be determined. In this study, by comparing the effects of irbesartan and hydralazine on DS rats, we obtained evidence that DS rats, at the stage of marked cardiac hypertrophy and fibrosis, had significant LV ischemia as a result of decreased capillary density. This decrease was mediated by angiotensin II and was independent of blood pressure. Of note, angiotensin II-induced cardiac ischemia in DS rats was associated with increased endothelial apoptosis, reduced LV VEGF expression and increased LV ASK1 activation.

Accumulating evidence indicates that oxidative stress has a key role in the pathophysiology of heart failure ${ }^{18-20}$ Moreover, angiotensin II is well known to induce cardiovascular oxidative stress, which has a pivotal role in the mechanism of angiotensin II-induced cardiac injury. ${ }^{18-20}$ Therefore, in this study, we also investigated the effects of tempol, a superoxide dismutase mimetic, in DS rats to elucidate the contribution of oxidative stress to cardiac injury. We found that tempol had a similar role as irbesartan in the attenuation of LV oxidative stress, which then prevented cardiac hypertrophy, fibrosis, abnormalities in cardiac capillary density and endothelial apoptosis, which is mediated by VEGF and ASK1 in DS rats. These results indicate the critical role of oxidative stress in cardiac injury. Together with the findings that both tempol and irbesartan attenuated cardiac oxidative stress in DS rats, our present findings show that the protective effects of irbesartan against cardiac injury are mainly mediated by the attenuation of oxidative stress.

The present observations showed that increased LV endothelial apoptosis in DS rats with significant cardiac hypertrophy could be attributed to oxidative stress. ASK1 is known to be one of the key molecules that is involved in oxidative stress-induced cellular apoptosis. ${ }^{14,21}$ These findings encouraged us to determine the role of ASK1 and VEGF in oxidative stress-induced endothelial apoptosis. Using cultured endothelial cells from wild-type and ASK1-deficient mice, we found that ASK1 deficiencies significantly attenuated $\mathrm{H}_{2} \mathrm{O}_{2}$-induced endothelial apoptosis, demonstrating the important role of ASK1 in oxidative stress-induced endothelial apoptosis. Interestingly, VEGF treatment mimicked the effect of ASK1 deficiency, as shown by the suppression of endothelial apoptosis by VEGF. Furthermore, this effect of VEGF was associated with a significant inhibition of oxidative stress-induced endothelial ASK1 activation. This suppression of ASK1 activation by VEGF may be due to the attenuation of endothelial oxidative stress by VEGF. Our observations provide the first evidence that VEGF prevents oxidative stress-induced endothelial apoptosis by suppressing ASK1.

\section{Study limitations}

Several study limitations should be considered in this study. First, we found no significant difference in cardiac phospho-eNOS and total eNOS among all groups of DS rats, thereby providing no evidence for the involvement of eNOS in cardiac injury. However, the potential role of eNOS in cardiac injury cannot be completely ruled out because eNOS is a major source of oxidative stress. In addition, VEGF can phosphorylate eNOS. Second, in this study, we did not examine the effect of each drug treatment on hemodynamics in DS rats. Therefore, we could not determine whether the abnormal changes in cardiac 
VEGF and ASK1 observed in this study were associated with the transition from compensated to decompensated cardiac hypertrophy. Third, the blood pressure measurements were performed by the tailcuff method instead of a direct method because measurement of a large number of rats was needed for this study. Fourth, the detailed mechanisms underlying the suppression of ASK1 activation by VEGF remain undefined. Finally, in this study, the involvement of ASK1 in the regulation of VEGF expression was not defined. Further research is necessary to address these key issues.

In conclusion, in DS rats with significant cardiac hypertrophy and fibrosis, angiotensin II decreased myocardial VEGF and activated ASK1 via oxidative stress. These detrimental effects of angiotensin II caused endothelial apoptosis, thereby reducing capillary density and causing subsequent myocardial ischemia. Thus, this study provides a novel mechanism responsible for angiotensin II-induced cardiac injury. However, further research is needed to elucidate whether our present findings contribute to the understanding of the transition from compensated to decompensated cardiac hypertrophy in hypertensive rats.

\section{CONFLICT OF INTEREST}

The authors declare no conflict of interest.

\section{ACKNOWLEDGEMENTS}

This work was supported in part by Grants-in-Aid for Scientific Research from the Ministry of Education, Culture, Sports, Science, and Technology.

1 Kim S, Iwao H. Molecular and cellular mechanisms of angiotensin ii-mediated cardiovascular and renal diseases. Pharmacol Rev 2000; 52: 11-34.

2 Kim S, Yoshiyama M, Izumi Y, Kawano H, Kimoto M, Zhan Y, Iwao H. Effects of combination of ace inhibitor and angiotensin receptor blocker on cardiac remodeling, cardiac function, and survival in rat heart failure. Circulation 2001; 103: 148-154.

3 Yamamoto E, Kataoka K, Shintaku H, Yamashita T, Tokutomi Y, Dong YF, Matsuba S, Ichijo H, Ogawa H, Kim-Mitsuyama S. Novel mechanism and role of angiotensin ii induced vascular endothelial injury in hypertensive diastolic heart failure. Arterioscler Thromb Vasc Biol 2007; 27: 2569-2575.

4 Yamamoto E, Kataoka K, Yamashita T, Tokutomi Y, Dong YF, Matsuba S, Ogawa H, Kim-Mitsuyama S. Role of xanthine oxidoreductase in the reversal of diastolic heart failure by candesartan in the salt-sensitive hypertensive rat. Hypertension 2007; 50: 657-662.

5 Mann DL, Bristow MR. Mechanisms and models in heart failure: the biomechanical model and beyond. Circulation 2005; 111: 2837-2849.

6 Yusuf S, Pfeffer MA, Swedberg K, Granger CB, Held P, McMurray JJ, Michelson EL, Olofsson B, Ostergren J. Effects of candesartan in patients with chronic heart failure and preserved left-ventricular ejection fraction: The charm-preserved trial. Lancet 2003; 362: 777-781.

7 Ferrara N. Vascular endothelial growth factor: Basic science and clinical progress. Endocr Rev 2004; 25: 581-611.

8 Hudlicka O, Brown M, Egginton S. Angiogenesis in skeletal and cardiac muscle. Physiol Rev 1992; 72: 369-417.

9 Sano M, Minamino T, Toko $\mathrm{H}$, Miyauchi $\mathrm{H}$, Orimo M, Qin $\mathrm{Y}$, Akazawa $\mathrm{H}$, Tateno $\mathrm{K}$, Kayama Y, Harada M, Shimizu I, Asahara T, Hamada H, Tomita S, Molkentin JD, Zou Y, Komuro I. P53-induced inhibition of hif-1 causes cardiac dysfunction during pressure overload. Nature 2007; 446: 444-448.

10 Shiojima I, Sato K, Izumiya Y, Schiekofer S, Ito M, Liao R, Colucci WS, Walsh K. Disruption of coordinated cardiac hypertrophy and angiogenesis contributes to the transition to heart failure. J Clin Invest 2005; 115: 2108-2118.

11 Doi R, Masuyama T, Yamamoto K, Doi Y, Mano T, Sakata Y, Ono K, Kuzuya T, Hirota S, Koyama T, Miwa T, Hori M. Development of different phenotypes of hypertensive heart failure: Systolic versus diastolic failure in dahl salt-sensitive rats. J Hypertens 2000; 18: 111-120.

12 Yamamoto E, Yamashita T, Tanaka T, Kataoka K, Tokutomi Y, Lai ZF, Dong YF, Matsuba S, Ogawa H, Kim-Mitsuyama S. Pravastatin enhances beneficial effects of olmesartan on vascular injury of salt-sensitive hypertensive rats, via pleiotropic effects. Arterioscler Thromb Vasc Biol 2007; 27: 556-563.

13 Nakamura T, Yamamoto E, Kataoka K, Yamashita T, Tokutomi Y, Dong YF, Matsuba S, Ogawa H, Kim-Mitsuyama S. Beneficial effects of pioglitazone on hypertensive cardiovascular injury are enhanced by combination with candesartan. Hypertension 2008; 51: 296-301.

14 Ichijo H, Nishida E, Irie K, ten Dijke P, Saitoh M, Moriguchi T, Takagi M, Matsumoto K, Miyazono K, Gotoh Y. Induction of apoptosis by ask1, a mammalian mapkkk that activates sapk/jnk and p38 signaling pathways. Science 1997; 275: 90-94.

15 Tobiume K, Matsuzawa A, Takahashi T, Nishitoh H, Morita K, Takeda K, Minowa O, Miyazono K, Noda T, Ichijo H. Ask1 is required for sustained activations of jnk/p38 map kinases and apoptosis. EMBO Rep 2001; 2: 222-228.

16 Yamamoto E, Lai ZF, Yamashita T, Tanaka T, Kataoka K, Tokutomi Y, Ito T, Ogawa H, Kim-Mitsuyama S. Enhancement of cardiac oxidative stress by tachycardia and its critical role in cardiac hypertrophy and fibrosis. J Hypertens 2006; 24: 2057-2069.

17 Izumiya Y, Shiojima I, Sato K, Sawyer DB, Colucci WS, Walsh K. Vascular endothelial growth factor blockade promotes the transition from compensatory cardiac hypertrophy to failure in response to pressure overload. Hypertension 2006; 47: 887-893.

18 Cai H, Harrison DG. Endothelial dysfunction in cardiovascular diseases: The role of oxidant stress. Circ Res 2000; 87: 840-844.

19 Griendling KK, Sorescu D, Ushio-Fukai M. Nad(p)h oxidase: role in cardiovascular biology and disease. Circ Res 2000; 86: 494-501.

20 Takimoto E, Kass DA. Role of oxidative stress in cardiac hypertrophy and remodeling. Hypertension 2007; 49: 241-248.

21 Matsukawa J, Matsuzawa A, Takeda K, Ichijo H. The ask1-map kinase cascades in mammalian stress response. J Biochem 2004; 136: 261-265. 